\title{
6. SIMULTANEOUS RADAR AND OPTICAL OBSERVATION OF METEORS AT OND REJOV IN 1962
}

\author{
J. Grygar, L. Kohoutek, Z. Plavcová \\ (Astronomical Institute of the Czechoslovak Academy of Sciences, Ondřejov and Prague)
}

\section{Observations}

A program of simultaneous observation of meteors was organized in the period between August 25 and September 3, 1962 at the Ondřejov Observatory. Experienced amateur astronomers, 37 in number, took part in the visual and telescopic observation of meteors according to the following scheme: four visual observers surveyed an area, the centre of which coincided with the axis of the antenna diagram of the Ondřejov meteor radar: elevation $45^{\circ}$, south azimuth. 24 observers used $10 \times 80$ binoculars with a field of view of $7.3^{\circ}$ diameter and kept under control three zones in the altitudes of $30^{\circ}, 45^{\circ}$, and $60^{\circ}$ above the horizon. In each zone, there were four azimuths of the centres of the fields of view, namely $349.5^{\circ}, 356.5^{\circ}, 3.5^{\circ}$ and $10.5^{\circ}$; each of these areas being observed by two observers. All standard data of meteor statistics were recorded. Time recording was doubled: recorders read the time indicated by light signals on a chronometer, and independently the time was indicated on a film moving in synchronization with the radar recording film. Thus an accuracy in time recording of $\pm 0.5 \mathrm{sec}$ was guaranteed. Unfortunately, the motor of the time camera sometimes caused interference of variable intensity unfavourably affecting the radar data, particularly the radar hourly rates.

The meteor radar worked on the frequency of $37.5 \mathrm{Mc} / \mathrm{sec}$ with the peak power of $20 \mathrm{~kW}$. The repetition frequency was 500 pulses of $10 \mu \mathrm{sec}$ duration per second. For each radar echo, the time of incidence, range with accuracy to $\pm 5 \mathrm{~km}$, duration, and maximum width of the echo were read out from the film records.

Simultaneous observations were carried out on 8 nights, corresponding to $28^{\mathrm{h}} 40^{\mathrm{m}}$ of net time. One third of the time was, however, lost due to the strong interference mentioned above. A survey of the material obtained is given in Table 1, where $n_{\mathrm{T}}$ is number of telescopic meteors in altitude zones $30^{\circ}, 45^{\circ}$, and $60^{\circ}$, and $n_{\mathrm{V}}, n_{\mathrm{R}}$, numbers of visual and radar meteors, respectively.

\section{Hourly Rates}

Standard hourly rates with no special correction for limiting magnitude and Kresák and Millman (eds.), Physics and Dynamics of Meteors, 63-69. O I.A.U. 


\section{Table 1}

\section{Observational results}

\begin{tabular}{|c|c|c|c|c|c|c|}
\hline \multirow[b]{2}{*}{ Date } & \multirow[b]{2}{*}{ Time } & \multicolumn{3}{|c|}{$n_{\mathrm{T}}$} & \multirow[t]{2}{*}{$n_{\curlyvee}$} & \multirow[t]{2}{*}{$n_{\mathrm{R}}$} \\
\hline & & $30^{\circ}$ & $45^{\circ}$ & $60^{\circ}$ & & \\
\hline $25 / 26$ & $4^{\mathrm{h}} 12^{\mathrm{m}}$ & 270 & 373 & 271 & 257 & 298 \\
\hline $26 / 27$ & 520 & 358 & 380 & 234 & 342 & 503 \\
\hline $27 / 28$ & 125 & 77 & 80 & 48 & 52 & 131 \\
\hline $28 / 29$ & 308 & 185 & 213 & 185 & 180 & 240 \\
\hline $30 / 31$ & 550 & 245 & 321 & 234 & $360^{\mathrm{a}}$ & 543 \\
\hline 31/Sept. 1 & 345 & 178 & 257 & 121 & $158^{\mathrm{a}}$ & 144 \\
\hline $1 / 2$ & 500 & 250 & 378 & 205 & 238 & $134^{b}$ \\
\hline $2 / 3$ & 410 & 195 & 263 & 190 & 205 & $235^{b}$ \\
\hline & $32^{\mathrm{h}} 50^{\mathrm{m}}$ & 1758 & 2265 & 1488 & 1792 & 2228 \\
\hline
\end{tabular}

a cloud interference.

b radar coverage incomplete.

meteorological conditions were calculated separately for telescopic, visual (both per single observer), and radar meteors. If cloud or electrical interference were too severe, such intervals were simply not taken into account. The hourly rates for 7 nights are summarized in Table 2, where $f$ with the proper subscript refers to hourly rates of

Table 2

Hourly rates

\begin{tabular}{rrrr}
\multicolumn{1}{c}{ Date } & $f_{\mathrm{T}}$ & \multicolumn{1}{c}{$f_{\mathrm{V}}$} & $f_{\mathrm{R}}$ \\
Aug. $25 / 26$ & $9 \cdot 2$ & $15 \cdot 2$ & $68 \cdot 8$ \\
$26 / 27$ & $8 \cdot 2$ & $16 \cdot 1$ & $94 \cdot 7$ \\
$27 / 28$ & $7 \cdot 1$ & $9 \cdot 3$ & $92 \cdot 8$ \\
$28 / 29$ & $8 \cdot 5$ & $14 \cdot 4$ & $72 \cdot 5$ \\
$30 / 31$ & $6 \cdot 2$ & $20 \cdot 3$ & $93 \cdot 0$ \\
Sept. $1 / 2$ & $7 \cdot 8$ & 11.9 & $75 \cdot 0$ \\
$2 / 3$ & 9.9 & $12 \cdot 3$ & $84 \cdot 0$
\end{tabular}

$T, V$ and $R$ meteors. Hourly rates for telescopic meteors are fairly constant while radar and visual data vary considerably. The pattern of the variations, however, shows a rather poor mutual correlation.

The diurnal variation was computed for 1-hour intervals between $21^{\text {h }}$ and $3^{\text {h }}$ of local time. Table 3 indicates that there is almost no variation for telescopic meteors, and this remains true even if we treat the different altitude zones separately. The visual and radar observations exhibit an almost parallel increase of hourly rates in the ratio of about 1:2. By extrapolation we can estimate that the overall diurnal variation of the visual and radar hourly rates was about 1:3. This is a rather low 
Table 3

\section{Diurnal variation}

\begin{tabular}{lllr}
$T(\mathrm{CET})$ & $f_{\mathrm{T}}$ & $f_{\mathrm{V}}$ & \multicolumn{1}{c}{$f_{\mathrm{K}}$} \\
$21^{\mathrm{h}}-22^{\mathrm{h}}$ & $7 \cdot 8$ & $11 \cdot 6$ & 66 \\
$22-23$ & $7 \cdot 4$ & 12.5 & 69 \\
$23-24$ & $7 \cdot 4$ & $10 \cdot 8$ & 87 \\
$24-01$ & $8 \cdot 3$ & $14 \cdot 3$ & 97 \\
$01-02$ & $8 \cdot 2$ & $16 \cdot 4$ & 107 \\
$02-03$ & $8 \cdot 3$ & $23 \cdot 5$ & 118
\end{tabular}

value for radar meteors. We believe that the low ratio was caused by the orientation of the radar antenna towards the south. The sharp distinction between the character of the diurnal variation found for the radar-visual and telescopic meteors, respectively, is so remarkable that we intend to study this effect at another Ondřejov meteor expedition.

\section{Luminosity Function}

The true number of visual meteors was found by using Kvíz's (1958) probability formula. In Table $\dot{4}$ we present the magnitude distribution of meteors; $n$ is the apparent number of meteors, $p$ the probability of perception, and $N$ the true number of meteors within a given magnitude. In Table 5 the quantities $N_{0}^{(5)}, N_{k}^{(5)}$ and $N_{k}^{+(5)}$ are the hourly numbers of meteors up to $5^{\mathrm{m}}$ per square degree, per square kilometer and per square kilometer in zenith, respectively. Table 5 also contains the respective data for telescopic meteors in different altitude zones as well as the $\kappa$ values. It is remarkable that the average $N_{k}^{+(5)}$ value derived from telescopic observation $(5 \cdot 7 \times$ $10^{-3} \mathrm{met} / \mathrm{km}^{2} /$ hour $)$ is in close agreement with the visual value $\left(5.6 \times 10^{-3} \mathrm{met} / \mathrm{km}^{2} /\right.$

Table 4

Luminosity function of visual meteors

$\begin{array}{lrcc}m & n & p & N \\ \leqslant 0 & 91 & 0.756 & 30.5 \\ 0.5 & 7 & (0 \cdot 221) & 7.9 \\ 1 & 54 & 0.793 & 17.0 \\ 1.5 & 87 & 0 \cdot 747 & 29 \cdot 1 \\ 2 & 141 & 0.579 & 60.7 \\ 2 \cdot 5 & 154 & 0.483 & 79.6 \\ 3 & 296 & 0.461 & 160.5 \\ 3 \cdot 5 & 254 & 0.337 & 188.3 \\ 4 & 145 & 0.182 & 199.5 \\ 4 \cdot 5 & 130 & 0 \cdot 053 & 613 \\ 5 & 104 & 0.033 & 789 \\ \geqslant 5.5 & 14 & - & -\end{array}$


Table 5

\section{Parameters of the luminosity function}

Telescopic

Visual

$\begin{array}{lc}\text { Zone } & +30 \\ & \\ N_{o^{(5)}} & 2.60 \times 10^{-2} \\ N_{k^{(5)}} & 1.07 \times 10^{-3} \\ N_{k}(5) & 7.66 \times 10^{-3} \\ \kappa & 4.66\end{array}$

$+45$

$+60$

Average

$$
\begin{gathered}
1.81 \times 10^{-2} \\
2.10 \times 10^{-3} \\
5.15 \times 10^{-3} \\
5.60
\end{gathered}
$$$$
1.50 \times 10^{-2}
$$$$
1.97 \times 10^{-2}
$$$$
3.14 \times 10^{-3}
$$$$
4.34 \times 10^{-3}
$$$$
3 \cdot 15
$$$$
\begin{gathered}
2.10 \times 10^{-3} \\
5.72 \times 10^{-3} \\
4.47
\end{gathered}
$$

$2.98 \times 10^{-2}$

$3.45 \times 10^{-3}$

$4 \cdot 47$

$5.56 \times 10^{-3}$

2.50

hour). The $\kappa$ value for telescopic meteors, equal to $4 \cdot 5$, is reasonably higher than the visual $\kappa=2.5$ as one should expect.

The radar data are shown in Figure 1, where $T_{D}$ denotes the echo duration while $N$ is the cumulative number of echoes. From the total of 484 echoes longer than $0.3 \mathrm{sec}$ we derived $s=2 \cdot 33$. The duration was corrected for the width of the echo, with no regard to the attachment of electrons to neutral particles. All echoes were in the time interval from 0.4 to $6.3 \mathrm{sec}$, which corresponds to the magnitude interval of $0 \cdot 4^{\mathrm{m}}-3 \cdot 4^{\mathrm{m}}$. The derived $s$ value corresponds to the slope $\kappa=3 \cdot 40$, which lies in the middle between the $\kappa$ slopes for visual and telescopic meteors.

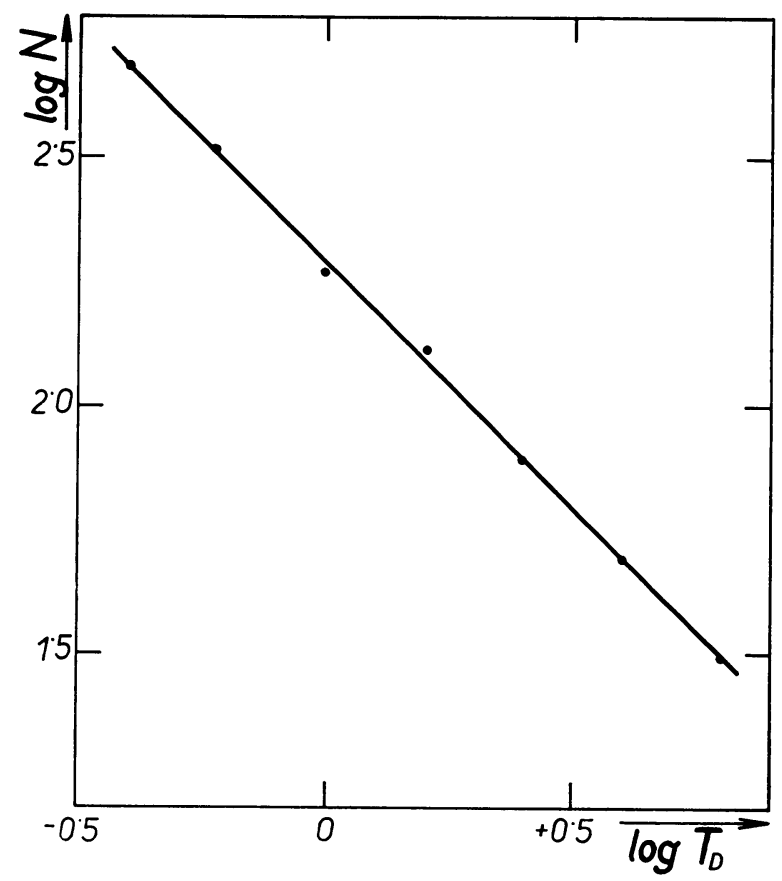

FIG. 1. The relationship between echo duration, $T_{D}$, and cumulative number of echoes, $N$. 
We also have at our disposal 2846 echoes from underdense trails. For this material the magnitudes were derived by the method described by Hajduk (1967). Thus we have $\kappa=3.75$ for the magnitude interval of $0.1-6^{\mathrm{m}} \cdot 2$, under the assumption that the transition between overdense and underdense trails occurs at $\alpha=2.4 \times 10^{14}$ electrons $/ \mathrm{m}$.

\section{Radar and Optical Coincidences}

Two criteria were used when looking for common visual and radar meteors. The first one was time coincidence and the second one the height of a meteor in the Earth's atmosphere. For each visual meteor we have looked over all radar echoes within the time interval of $\pm 20 \mathrm{sec}$ and the height range $85-100 \mathrm{~km}$. The results are presented, in the form of a histogram, in Figure 2. We observe that often the visual time is just $1 \mathrm{sec}$ late in comparison with the time of the radar echo, which is easily understandable as the result of personal equation of optical observers. Dashed parts of the histogram correspond to somewhat uncertain visual time data. In the upper part of Figure 2 the coincidences for radar echoes of longer $(\geqslant 0.5 \mathrm{sec})$ duration are
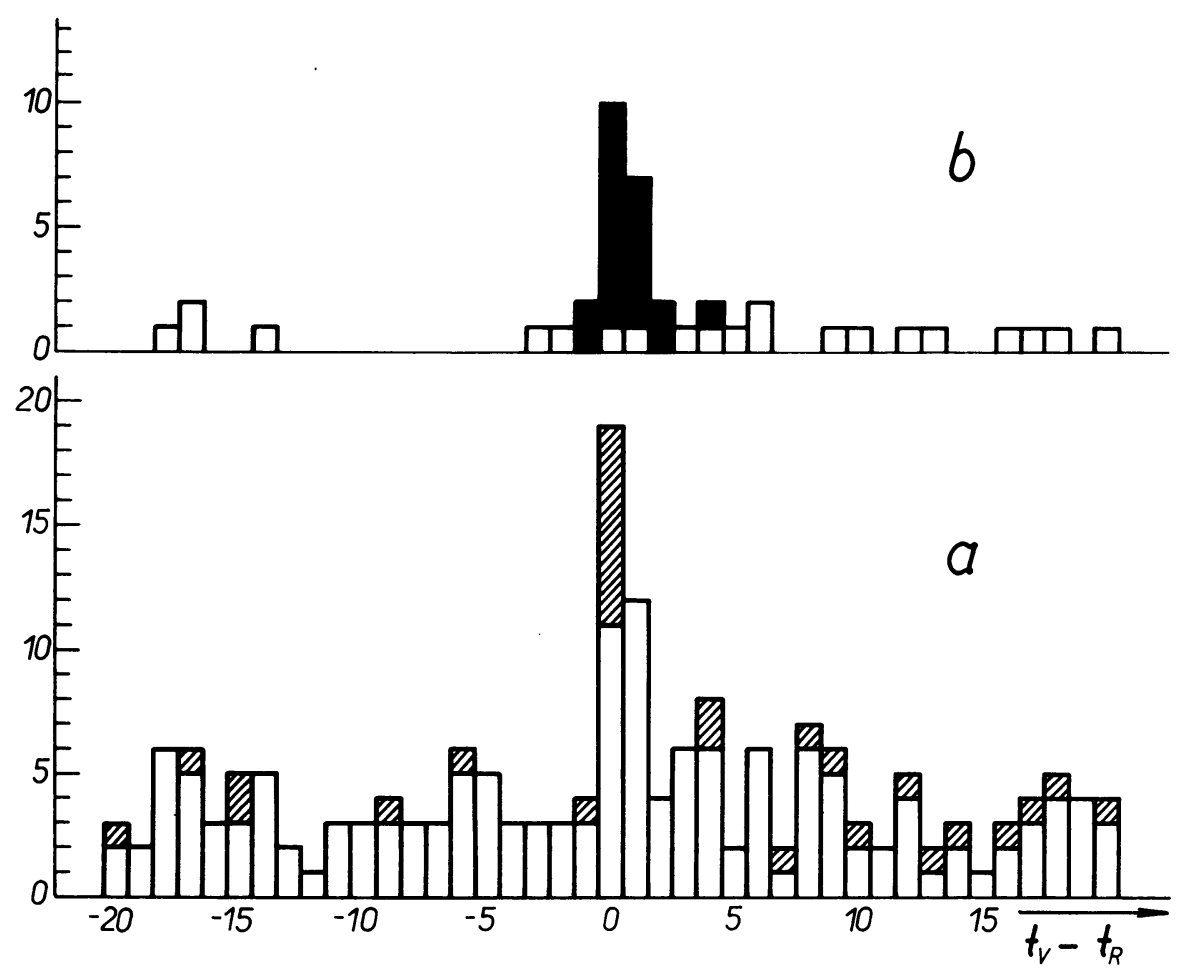

FIG. 2. Time coincidences of radar $\left(t_{\mathrm{R}}\right)$ and visual $\left(t_{\mathrm{V}}\right)$ data. (a) All coincidences; dashed areas correspond to somewhat uncertain $t_{\mathrm{V}}$ data. (b) Coincidences for radar echoes of longer duration $(\geqslant 0.5 \mathrm{sec})$. Real meteor coincidences are drawn in full black. 
plotted. Again the most frequent differences are 0 and $+1 \mathrm{sec}$. Real coincidences are drawn in full black. There were just 20 cases of these. Visual magnitudes of these meteors were corrected to $100 \mathrm{~km}$ distance, and such corrected absolute visual magnitudes are plotted in Figure 3 against the echo duration $T_{D}$. In this way we have derived the formula

$$
\begin{aligned}
M= & 42 \cdot 4-2 \cdot 63 \log \alpha, \\
& \pm 7 \cdot 7 \quad \pm .48
\end{aligned}
$$

valid for overdense trails.

All meteors with the echo duration of 12 to $29 \mathrm{sec}$ (absolute visual magnitude interval $-1^{\mathrm{M}}$ to $0^{\mathrm{M}}$ ) were recorded by visual observers. Only $54 \%$ of echoes with

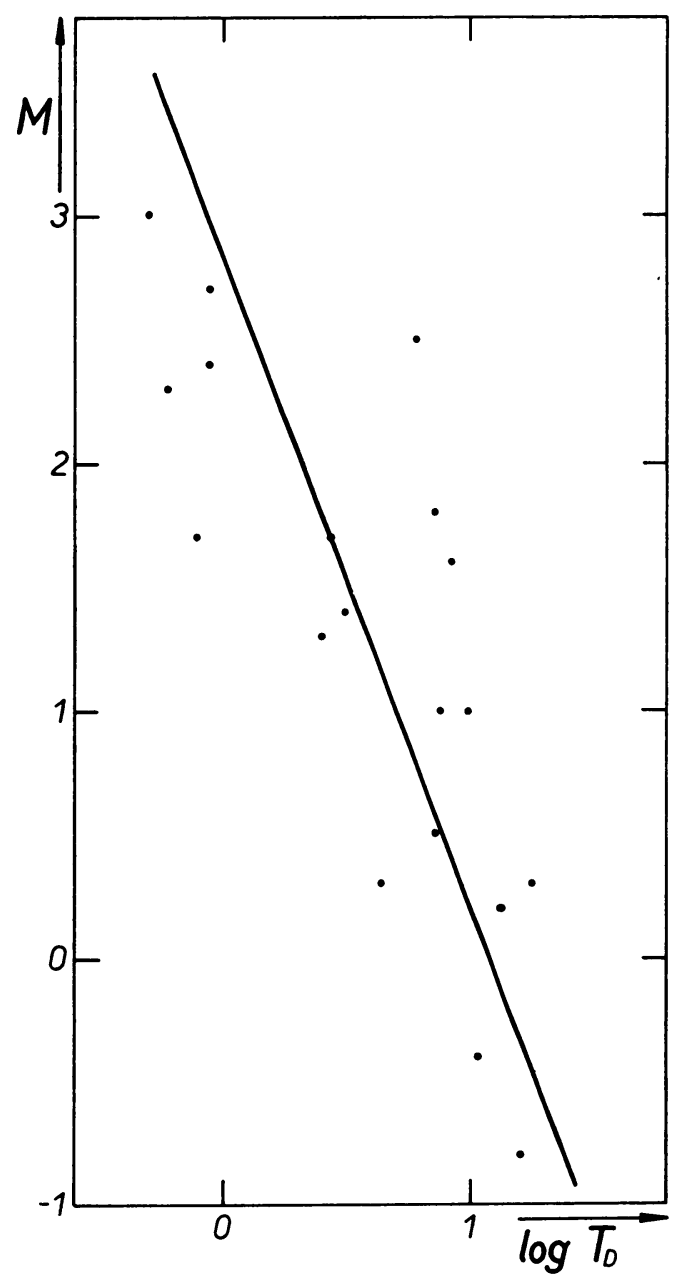

FIG. 3. The relationship between meteor echo duration $T_{\mathrm{D}}$ and absolute visual magnitude $M$. 
duration of 5-12 sec were similarly recorded. With the decreasing echo duration the number of coincidences naturally becomes smaller, and for echoes shorter than 0.5 sec no reliable coincidences were found at all. On the contrary, only $12 \%$ of visual meteors in the magnitude interval of $1^{\mathrm{m}}$ to $3^{\mathrm{m}}$ were simultaneously recorded by radar. We see that the selection effects for radar and visual meteors are working in completely different ways.

The average angular length for all visual meteors was $12 \cdot 2^{\circ}$, while for meteors simultaneously observed by radar the value was $15.4^{\circ}$. This was to be expected since longer meteors are, on the average, brighter and thus the chance of simultaneous observation increases. On the other hand, the average angular velocity is exactly the same for all visual meteors as for the set of meteors simultaneously observed by radar. This is rather surprising since one would expect that the radar prefers faster meteors at greater distances from the radiant.

In spite of great effort, we were unable to find any single positive coincidence of a radar and a telescopic meteor. The chance coincidences were so frequent that it seems impossible to find real coincidences unless some additional criteria for common meteors are discovered.

\section{References}

Hajduk, A. (1967) Ph.D. Thesis, Bratislava (unpublished).

Kvíz, Z. (1958) Bull. astr. Inst. Csl., 9, 70.

\section{DISCUSSION}

Millman: Data from the Ottawa radio-meteor program lends support to the great difference in the selection effects between radar and visual observations respectively. Many fairly bright visual meteors have no radar echoes and many strong radar echoes have no corresponding visual meteors. 